\title{
Caracterização estrutural por difração de raios $X$ de alta resolução de SiAIONs sinterizados com diferentes aditivos
}

\author{
(Structural characterization by high-resolution X-ray \\ diffraction of SiAlONs sintered with different additives)
}

\author{
C. Santos ${ }^{1}$, P. A. Suzuki ${ }^{1}$, K. Strecker ${ }^{1}$, S. Kycia ${ }^{2,3}$, C. R. M. Silva ${ }^{4}$ \\ ${ }^{1}$ FAENQUIL-DEMAR, C.P.116, Lorena, SP, Brasil 12600-970 \\ ${ }^{2}$ LNLS, Campinas, SP, Brasil \\ ${ }^{3}$ Phys. Dept., University of Gwelph, Gwelph, Canadá \\ ${ }^{4}$ CTA-IAE/AMR, S. José dos Campos, SP, Brasil \\ claudinei@ppgem.faenquil.br
}

\begin{abstract}
Resumo
A técnica de difração de raios $\mathrm{X}$ de alta resolução foi utilizada para a caracterização estrutural da solução sólida, formada a partir de nitreto de silício $\left(\mathrm{Si}_{3} \mathrm{~N}_{4}\right)$, denominada $\alpha$-SiAlON. Cerâmicas de $\alpha$-SiAlON foram produzidas utilizando aditivos à base de $\mathrm{AlN}_{2} \mathrm{Y}_{2} \mathrm{O}_{3}$ ou $\mathrm{AlN}-\mathrm{CRE} \mathrm{O}_{2}$. $\mathrm{O}$ óxido misto, $\mathrm{CRE}_{2} \mathrm{O}_{3}$, é uma solução sólida formada por $\mathrm{Y}_{2} \mathrm{O}_{3}$ e óxidos de terras raras. Os resultados mostraram a formação de solução sólida nos materiais sinterizados e não foi detectada segregação de óxidos utilizados como aditivos de sinterização. A similaridade das propriedades estruturais, morfológicas e mecânicas entre $\mathrm{SiAlONs}$ produzidos com aditivos contendo $\mathrm{Y}_{2} \mathrm{O}_{3}$ ou $\mathrm{CRE}_{2} \mathrm{O}_{3}$ indica a possibilidade de substituição do $\mathrm{Y}_{2} \mathrm{O}_{3}$ por $\mathrm{CRE}_{2} \mathrm{O}_{3}$ na produção de $\alpha$-SiAlONs por um custo mais baixo.

Palavras-chave: SiAlON, óxido misto, difração de raios $\mathrm{X}$ de alta resolução, luz síncrotron, refinamento de estrutura, método de Rietveld.
\end{abstract}

Abstract

Solid solutions formed by silicon nitride, known as SiAlONs, were prepared using different additives, such as $\mathrm{AlN}-\mathrm{Y}_{2} \mathrm{O}_{3}$ or $\mathrm{AlN}-\mathrm{CRE} \mathrm{O}_{3}$. The mixed oxide $\mathrm{CRE}_{2} \mathrm{O}_{3}$ is a solid solution formed by $\mathrm{Y}_{2} \mathrm{O}_{3}$ and rare-earth oxides. The high-resolution $X$-ray diffraction results of the sintered material show the formation of a solid solution. No segregation of second phases was detected. The similarities of structural, morphological and mechanical properties among SiAlONs prepared from different additives containing $\mathrm{Y}_{2} \mathrm{O}_{3}$ or $\mathrm{CRE}_{2} \mathrm{O}_{3}$ show the possibility of the production of $\alpha$-SiAlON using $C R E_{2} \mathrm{O}_{3}$ rather than $\mathrm{Y}_{2} \mathrm{O}_{3}$ as additive at lower cost.

Keywords: SiAlON, mixed oxide, high-resolution X-ray diffraction, synchrotron, structure refinement, Rietveld method.

\section{INTRODUÇÃO}

Cerâmicas estruturais à base de nitreto de silício $\left(\mathrm{Si}_{3} \mathrm{~N}_{4}\right)$ têm sido intensivamente investigadas devido às suas propriedades termomecânicas, tais como a alta resistência à fluência e estabilidade estrutural em temperaturas elevadas [1-3]. Um dos principais inconvenientes encontrados na sua fabricação é a dificuldade de densificação durante a etapa de sinterização. Dada esta dificuldade, faz-se necessária a utilização de aditivos que fundem em temperaturas inferiores às da sinterização, promovendo a formação de uma fase líquida que envolva as partículas do material base, molhando-as em condições favoráveis e facilitando os processos difusionais responsáveis pela redução da porosidade e transformação de fases durante o processo de sinterização $[3,4]$.

Como resultado da aplicação de tais aditivos, as principais propriedades de nitreto de silício em altas temperaturas tendem a sofrer degradação. Por outro lado, hoje é bem conhecido que a classe de soluções sólidas de $\mathrm{Si}_{3} \mathrm{~N}_{4}$, denominadas SiAlONs $(\alpha$ ou $\beta$ ), possui muitas vantagens em relação às cerâmicas de $\mathrm{Si}_{3} \mathrm{~N}_{4}$ tradicionais, devido à incorporação de grande parte dos elementos presentes nos aditivos na estrutura cristalina do $\mathrm{Si}_{3} \mathrm{~N}_{4}$ durante a sinterização. Assim, diminui-se a quantidade de aditivos, e conseqüentemente, melhora-se as propriedades mecânicas em temperaturas elevadas, principalmente, a resistência à oxidação e à fluência $[1,2,5,6]$.

O $\alpha$-SiAlON (ou $\alpha^{*}$ ), cuja fórmula química é $\mathrm{M}_{x} \mathrm{Si}_{12-(m+n)} \mathrm{Al}_{(m+n)} \mathrm{O}_{n} \mathrm{~N}_{16-n}$, com $\mathrm{M}$ representando o cátion metálico usado como estabilizador desta fase é uma solução sólida de $\alpha-\mathrm{Si}_{3} \mathrm{~N}_{4}$, onde $\mathrm{Si}$ e $\mathrm{N}$ são parcialmente substituídos por $\mathrm{Al}$ e $\mathrm{O}$, respectivamente. Para a formação deste material é necessário o uso de aditivos 
de sinterização ricos em $\mathrm{Al}$ e $\mathrm{O}$, além de átomos, tais como $\mathrm{Y}$ ou terras raras, que ocupem posições intersticiais na estrutura do $\alpha-\mathrm{Si}_{3} \mathrm{~N}_{4}$, promovendo sua estabilização em temperaturas elevadas [2, 4]. Esta substituição gera um material de dureza superior ao $\alpha-\mathrm{Si}_{3} \mathrm{~N}_{4}$ e com maior resistência à fluência e à oxidação, já que parte da fase líquida transiente formada durante o aquecimento, é consumida para a formação de solução sólida.

A utilização do aditivo $\mathrm{CRE}_{2} \mathrm{O}_{3}$ (óxido misto de ítrio e terras raras) fabricado na FAENQUIL demonstrou ser um potencial substituto ao $\mathrm{Y}_{2} \mathrm{O}_{3}$ como estabilizador do $\alpha-\mathrm{Si}_{3} \mathrm{~N}_{4}$ com propriedades físicas, químicas e mecânicas similares, e um custo $80 \%$ inferior ao $\mathrm{Y}_{2} \mathrm{O}_{3}$ puro [7]. Em trabalho anterior foi mostrado que este óxido misto de terras raras, composto majoritariamente de: $\mathrm{Y}_{2} \mathrm{O}_{3}, \mathrm{Yb}_{2} \mathrm{O}_{3}$, $\mathrm{Dy}_{2} \mathrm{O}_{3}$ e $\mathrm{Nd}_{2} \mathrm{O}_{3}$, forma uma solução sólida com o óxido majoritário, $\mathrm{Y}_{2} \mathrm{O}_{3}[8]$.

A difração de raios $X$ de alta resolução com uma fonte de luz síncrotron é uma técnica que oferece alta intensidade e alta resolução. É uma técnica apropriada para avaliar e comparar soluções sólidas, tais como os SiAlONs produzidos com $\mathrm{Y}_{2} \mathrm{O}_{3}$ puro ou com $\mathrm{CRE}_{2} \mathrm{O}_{3}$ como aditivos, pois pequenas variações na estrutura e possíveis segregações de óxidos de terras raras podem ser detectadas. Os objetivos deste trabalho foram: a verificação da formação da solução sólida e a determinação dos parâmetros estruturais de $\mathrm{SiAlONs}$ produzidos a partir de nitreto de silício e os aditivos $\mathrm{AlN}$ e $\mathrm{Y}_{2} \mathrm{O}_{3}$ ou AlN e $\mathrm{CRE}_{2} \mathrm{O}_{3}$ utilizando a difração de raios $\mathrm{X}$ de alta resolução. Os resultados são avaliados juntamente com os resultados publicados recentemente [8], permitindo compreender melhor as propriedades físicas, químicas e mecânicas apresentadas por estes materiais.

\section{MATERIAIS E MÉTODOS}

\section{Processamento}

Os corpos sinterizados foram obtidos a partir da mistura de pós contendo $\mathrm{Si}_{3} \mathrm{~N}_{4}$ (HCST), com $20 \%$ em volume de aditivos de: i) AlN (HCST) e $\mathrm{Y}_{2} \mathrm{O}_{3}$ (HCST) e ii) AlN (HCST) e $\mathrm{CRE}_{2} \mathrm{O}_{3}$, ambas com uma relação molar de 9:1 entre os aditivos AlN:Y ou AlN:(CRE) $)_{2}$. Estas misturas de pós foram denominadas SNYA20 e SNCA20, respectivamente, e suas composições são apresentadas na Tabela I.

Tabela I - Composição das misturas de pós. [Table I - Composition of the powder mixtures.]

\begin{tabular}{ccc}
\hline Componente & SNYA20 & SNCA20 \\
\hline $\mathrm{Si}_{3} \mathrm{~N}_{4}$ & 76,4 & 75,3 \\
$\mathrm{AlN}$ & 13,1 & 13,5 \\
$\mathrm{Y}_{2} \mathrm{O}_{3}$ & 10,5 & ----- \\
$\mathrm{CRE}_{2} \mathrm{O}_{3}$ & ---- & 11,2 \\
\hline
\end{tabular}

$\mathrm{O}$ óxido misto formado de ítria e de terras raras, $\mathrm{CRE}_{2} \mathrm{O}_{3}$, é uma solução sólida de óxido de ítrio e de terras raras produzido na FAENQUIL e possui características de sinterabilidade similares ao $\mathrm{Y}_{2} \mathrm{O}_{3}$ comercial e um custo de obtenção $80 \%$ inferior [7-11]. A composição química de $\mathrm{CRE}_{2} \mathrm{O}_{3}$ determinada por análise química está mostrada na Tabela II.

Tabela II - Composição do óxido misto $\left(\mathrm{CRE}_{2} \mathrm{O}_{3}\right)$ formado por óxido de ítrio e óxidos de terras raras.

[Table II - Composition of the $\mathrm{CRE}_{2} \mathrm{O}_{3}$ oxide composed by yttrium and rare earth oxides.]

\begin{tabular}{cccc}
\hline óxido & \% peso & óxido & \% peso \\
\hline $\mathrm{Y}_{2} \mathrm{O}_{3}$ & 44,60 & $\mathrm{Gd}_{2} \mathrm{O}_{3}$ & 1,17 \\
$\mathrm{Yb}_{3} \mathrm{O}_{3}$ & 19,71 & $\mathrm{~Tb}_{2} \mathrm{O}_{3}$ & 0,82 \\
$\mathrm{Er}_{2} \mathrm{O}_{3}$ & 13,94 & $\mathrm{Sm}_{2} \mathrm{O}_{3}$ & 0,38 \\
$\mathrm{Dy}_{2} \mathrm{O}_{3}$ & 10,25 & $\mathrm{Ce}_{2} \mathrm{O}_{3}$ & 0,09 \\
$\mathrm{Ho}_{2} \mathrm{O}_{3}$ & 3,27 & $\mathrm{Eu}_{2} \mathrm{O}_{3}$ & 0,02 \\
$\mathrm{Tm}_{2} \mathrm{O}_{3}$ & 2,83 & $\mathrm{Nd}_{2} \mathrm{O}_{3}$ & 0,12 \\
$\mathrm{Lu}_{2} \mathrm{O}_{3}$ & 2,62 & $\mathrm{La}_{2} \mathrm{O}_{3}$ & 0,01 \\
\hline
\end{tabular}

Os pós foram misturados e moídos em um moinho planetário, utilizando etanol como meio moedor por $2 \mathrm{~h}$. Após a moagem, as misturas de pós foram secadas a $100{ }^{\circ} \mathrm{C}$, por 12 $\mathrm{h}$, sendo em seguida peneiradas para a desaglomeração. As misturas foram submetidas à compactação por prensagem a frio em dois estágios: prensagem uniaxial a frio com $50 \mathrm{MPa}$ de pressão e subseqüente prensagem isostática a frio $(300 \mathrm{MPa})$. Os compactos foram submetidos a sinterização normal, em dois estágios: Aquecimento até $1750{ }^{\circ} \mathrm{C}$ com taxa de aquecimento de $25^{\circ} \mathrm{C} / \mathrm{min}$ à pressão de $0,1 \mathrm{MPa}$ em $\mathrm{N}_{2}$ e patamar de 30 min nesta temperatura. De 1750 a $1900^{\circ} \mathrm{C}$, os compactos foram aquecidos a uma taxa de $15^{\circ} \mathrm{C} / \mathrm{min}$, com pressão de $1,5 \mathrm{MPa}$ de $\mathrm{N}_{2}$ e patamar de $60 \mathrm{~min}$.

\section{Caracterizações}

As amostras sinterizadas foram fragmentadas, moídas e em seguida, peneiradas, passando o pó em uma peneira de $20 \mu \mathrm{m}$. Estes pós foram submetidos à difração de raios $\mathrm{X}$ de alta resolução com fonte de luz síncrotron. As medidas de difração de alta resolução foram realizadas no Laboratório Nacional de Luz Síncrotron (LNLS) em Campinas, SP, na linha de luz XRD1-D12A, em um difratômetro de múltiplos eixos Hubber. As medidas foram feitas em uma configuração de dois círculos concêntricos acoplados $(\theta-2 \theta)$, com incidência de um feixe monocromatizado de $10 \mathrm{keV}(\lambda=1,2398 \AA)$. Os pós foram pulverizados em um suporte cilíndrico $(10 \mathrm{~mm}$ de diâmetro e $2 \mathrm{~mm}$ de profundidade), mantido em rotação para promover a aleatoriedade dos planos cristalinos. O feixe difratado foi coletado por um cristal analisador de germânio (200) e detector de cintilação.

A simulação da estrutura cristalina foi feita utilizando o programa PowderCell [12]. O refinamento dos parâmetros 
da estrutura cristalina foi realizado pelo método de Rietveld usando o programa FullProf [13].

\section{RESULTADOS E DISCUSSÃO}

\section{Comparação entre nitreto de silício e SiAlON}

O pó de nitreto de silício comercial utilizado neste trabalho foi caracterizado por difração de alta resolução e está mostrada na Fig. 1a. Foram observadas as fases $\alpha-\mathrm{Si}_{3} \mathrm{~N}_{4}$ e $\beta-\mathrm{Si}_{3} \mathrm{~N}_{4}$, indicadas respectivamente por (1) e (2). Nenhuma

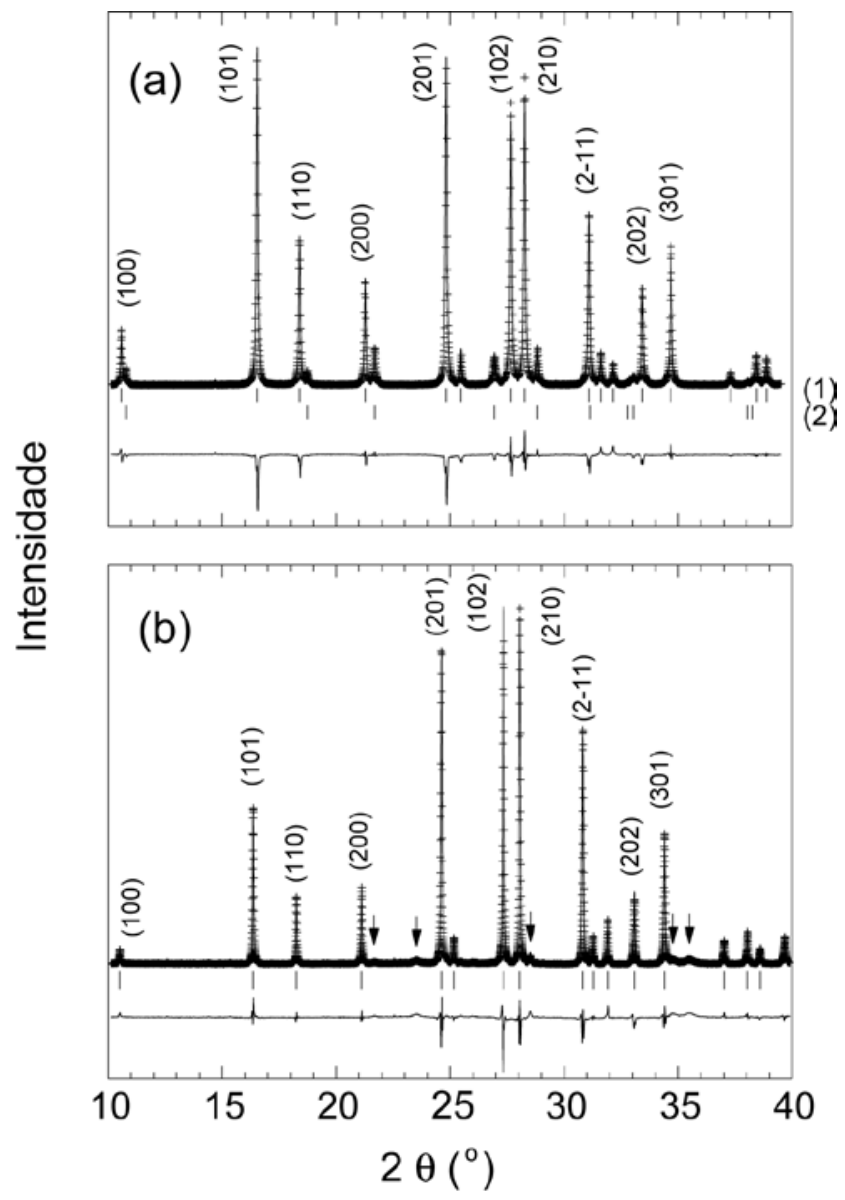

Figura 1: Resultados de refinamento pelo método de Rietveld dos difratogramas de alta resolução de: (a) nitreto de silício comercial e (b) SiAlON sinterizado com $\mathrm{Y}_{2} \mathrm{O}_{3}$ e AlN como aditivos. Os dados experimentais estão simbolizados por $(+)$. A linha contínua representa o difratograma calculado. As barras verticais indicam as posições das reflexões. (1) indica as posições das reflexões de $\alpha-\mathrm{Si}_{3} \mathrm{~N}_{4}$ e (2) indica as posições das reflexões de $\beta-\mathrm{Si}_{3} \mathrm{~N}_{4}$. Somente as reflexões mais intensas estão indexadas pelos índices de Miller. As reflexões não identificadas estão indicadas por setas. A linha contínua abaixo do difratograma representa a diferença entre o difratograma experimental e calculado.

[Figure 1: Rietveld analysis of high-resolution X-ray diffraction patterns of (a) commercial silicon nitride and (b) SiAlON sintered with $\mathrm{Y}_{2} \mathrm{O}_{3}$ and AlN sintering aids. (+): experimental; continuous line: calculated. (1) $\alpha-S i_{3} N_{4}$; (2) $\beta-S i_{3} N_{4}$. . outra fase foi detectada dentro do limite de detecção de $1 \%$. O refinamento de parâmetros estruturais pelo método de Rietveld foi feito baseado nos dados da literatura [14, 15]. Os parâmetros de rede refinados para as fases foram: $a=7,755(3) \AA$ e $c=5,6219(4) \AA$ para a fase $\alpha-\mathrm{Si}_{3} \mathrm{~N}_{4} \mathrm{e}$ $a=7,608(1) \AA$ e $c=2,9087(8) \AA$ para a fase $\beta-\mathrm{Si}_{3} \mathrm{~N}_{4}$. O refinamento pelo método de Rietveld indicou a presença de $92 \%$ da fase $\alpha-\mathrm{Si}_{3} \mathrm{~N}_{4}$ e $8 \%$ da fase $\beta-\mathrm{Si}_{3} \mathrm{~N}_{4}$.

$\mathrm{O} \alpha$-SiAlON formado a partir do nitreto de silício, AlN e $\mathrm{Y}_{2} \mathrm{O}_{3}$ está mostrado na Fig. 1b. Os parâmetros estruturais necessários para o refinamento pelo método de Rietveld

(a)

(b)

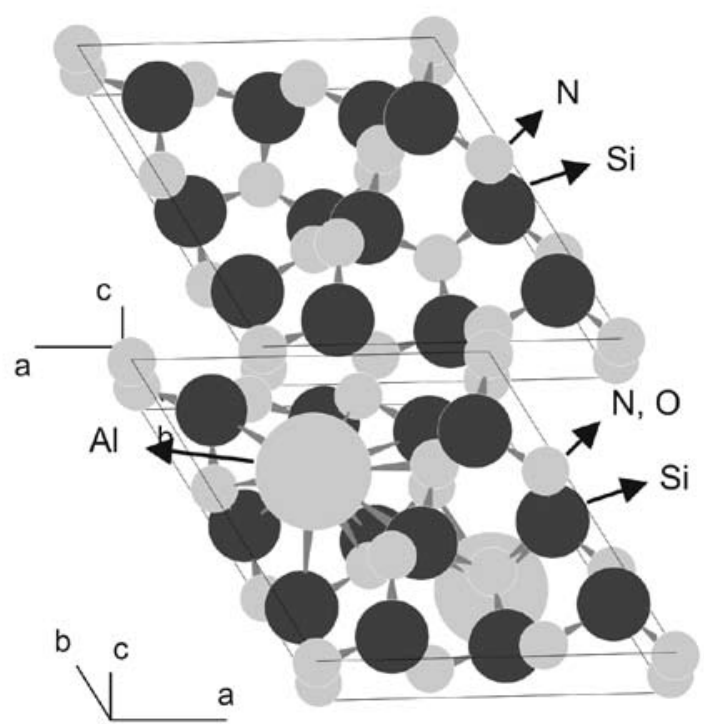

Figura 2: Modelos de estruturas cristalinas de: (a) $\alpha-\mathrm{Si}_{3} \mathrm{~N}_{4}$ e (b) $\alpha$-SiAlON.

[Figure 2: Models of crystalline structures: (a) $\alpha-\mathrm{Si}_{3} \mathrm{~N}_{4}$; (b) $\left.\mathrm{\beta}-\mathrm{Si}_{3} \mathrm{~N}_{4} \cdot\right]$

foram obtidos na literatura [16]. Os parâmetros de rede refinados para $\alpha$-SiAlON foram: $a=7,8126(1)$ $\AA$ e $c=5,6920(2) \AA$.

A Fig. 2 mostra os modelos de estruturas cristalinas simulados a partir de parâmetros estruturais de $\alpha-\mathrm{Si}_{3} \mathrm{~N}_{4}$ e $\alpha$-SiAlON. Pode-se observar que a estrutura cristalina de $\alpha$-SiAlON mantém a mesma simetria de $\alpha-\mathrm{Si}_{3} \mathrm{~N}_{4}$ diferindo em relação à estrutura de $\alpha-\mathrm{Si}_{3} \mathrm{~N}_{4}$ pelas posições intersticiais ocupadas pelo $\mathrm{Y}$ e substituição parcial de $\mathrm{O}$ por $\mathrm{N}$. A dissolução destes átomos em sítios intersticiais parece ser responsável pela expansão dos parâmetros de rede observados no $\alpha$-SiAlON em relação aos parâmetros de rede de $\alpha-\mathrm{Si}_{3} \mathrm{~N}_{4}$.

Análise dos óxidos $\mathrm{Y}_{2} \mathrm{O}_{3}$ e $\mathrm{CRE}_{2} \mathrm{O}_{3}$

Os pós dos óxidos $\mathrm{Y}_{2} \mathrm{O}_{3}$ e $\mathrm{CRE}_{2} \mathrm{O}_{3}$ foram analisados por meio de difração de raios $\mathrm{X}$ de alta resolução (não mostrado). Os resultados indicaram que o tipo de estrutura de $\mathrm{CRE}_{2} \mathrm{O}_{3}$ é similar ao de $\mathrm{Y}_{2} \mathrm{O}_{3}$ (rede cúbica, grupo espacial 
Ia-3), apresentando parâmetros de rede muito próximos. $\mathrm{O}$ parâmetro de rede determinado para $\mathrm{Y}_{2} \mathrm{O}_{3}$ foi $a=10,6080(5)$ $\AA$, enquanto que o parâmetro de rede determinado para $\mathrm{CRE}_{2} \mathrm{O}_{3}$ indicou o valor de $a=10,588(1) \AA ̊$. Esta diferença no parâmetro de rede $(\Delta a=0,020 \AA)$ foi atribuída às distorções causadas na rede pela ocupação substitucional de íons de $\mathrm{Yb}^{+3}, \mathrm{Er}^{+3}, \mathrm{Dy}^{+3}$, entre outros, em posições do $\mathrm{Y}^{+3}$ no óxido misto, alterando levemente o parâmetro de rede médio da estrutura. A largura a meia altura dos picos de $\mathrm{Y}_{2} \mathrm{O}_{3}$ e $\mathrm{CRE}_{2} \mathrm{O}_{3}$ apresentou diferença significativa. Enquanto a largura dos picos de $\mathrm{Y}_{2} \mathrm{O}_{3}$ foi próxima de $0,04^{\circ}$, a largura dos picos observada para $\mathrm{CRE}_{2} \mathrm{O}_{3}$ foi da ordem de $0,50^{\circ}$. As

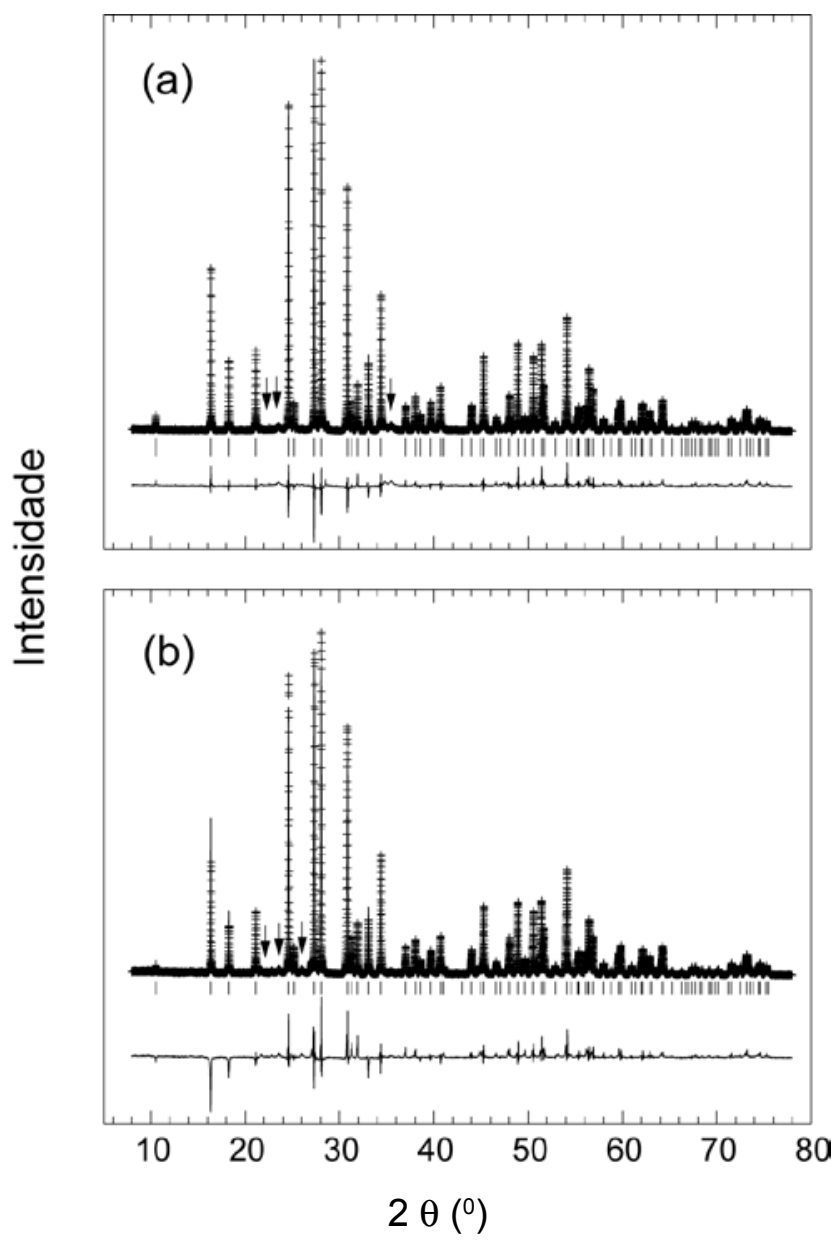

Figura 3: Resultados de refinamento pelo método de Rietveld dos difratogramas de alta resolução de: (a) $\alpha$-SiAlON sinterizados com $\mathrm{AlN}$ e $\mathrm{Y}_{2} \mathrm{O}_{3}$ e (b) $\alpha$-SiAlON sinterizados com AlN e CRE $\mathrm{O}_{3}$. Os dados experimentais estão simbolizados por: $(+)$. A linha contínua representa o difratograma calculado. As reflexões não identificadas estão indicadas por setas. As barras verticais indicam as posições das reflexões. A linha contínua abaixo do difratograma representa a diferença entre o difratograma experimental e calculado.

[Figure 3: Rietveld analysis of high-resolution X-ray diffraction patterns of (a) $\alpha-S i A l O N$ sintered with AlN and $\mathrm{Y}_{2} \mathrm{O}_{3}$ sintering aids and (b) $\alpha-S i A l O N$ sintered with AlN and $C R E_{2} O_{3}$ sintering aids. $(+)$ : experimental; continuous line: calculated.] intensidades das reflexões de $\mathrm{CRE}_{2} \mathrm{O}_{3}$ foram aproximadamente 8 vezes menores do que as intensidades das reflexões de $\mathrm{Y}_{2} \mathrm{O}_{3}$. A menor intensidade dos picos e a presença de reflexões largas indicam uma desordem na rede cristalina de $\mathrm{CRE}_{2} \mathrm{O}_{3}$ provocada provavelmente pelos íons substitucionais.

Comparação entre $\alpha-S i A l O N$ formado com $\mathrm{Y}_{2} \mathrm{O}_{3}$ ou $\mathrm{CRE}_{2} \mathrm{O}_{3}$ como aditivos

As amostras sinterizadas com $\mathrm{Y}_{2} \mathrm{O}_{3}$ (SNYA20) ou $\mathrm{CRE}_{2} \mathrm{O}_{3}$ (SNCA20) como aditivos foram submetidas a difração de raios X de alta resolução. A Fig. 3 apresenta os difratogramas e os resultados de refinamento pelo método de Rietveld destas amostras. As amostras são constituídas predominantemente por $\alpha$-SiAlON.As fases secundárias, não identificadas, apresentaram reflexões quase imperceptíveis, com intensidades inferiores a 3\%. Estas reflexões não são detectadas numa medida de difração convencional utilizando tubo de raios $\mathrm{X}$, devido a baixa intensidade. Não foram encontrados indícios de segregação de óxidos utilizados como aditivos.

Os difratogramas de $\alpha$-SiAlONs sinterizados com $\mathrm{Y}_{2} \mathrm{O}_{3}$ ou $\mathrm{CRE}_{2} \mathrm{O}_{3}$ apresentaram similaridade do ponto de vista da estrutura cristalina. Em ambos os difratogramas, as reflexões apresentam-se intensas e estreitas, indicando a cristalização da fase $\alpha$-SiAlON. As posições das reflexões foram praticamente asmesmas $\left(\Delta 2 \theta<0,01^{\circ}\right)$ nas duas amostras, independentemente do aditivo utilizado na sinterização. Isto indica que os parâmetros de rede dos $\alpha$-SiAlONs devem ser praticamente os mesmos, como serão mostrados a seguir. A largura dos picos também foi similar para SiAlONs estabilizados com $\mathrm{Y}_{2} \mathrm{O}_{3}\left(0,05^{\circ}\right)$ ou $\mathrm{CRE}_{2} \mathrm{O}_{3}\left(0,05^{\circ}\right)$. Entretanto, as intensidades das reflexões do $\alpha$-SiAlON sinterizado com $\mathrm{Y}_{2} \mathrm{O}_{3}$ foram em média 2 vezes maiores que as das reflexões do $\alpha$-SiAlON sinterizado com $\mathrm{CRE}_{2} \mathrm{O}_{3}$. Este resultado pode indicar uma cristalização um pouco menor do $\alpha$-SiAlON sinterizado com $\mathrm{CRE}_{2} \mathrm{O}_{3}$. É interessante notar que os $\alpha$-SiAlONs sinterizados

Tabela III - Parâmetros estruturais refinados de $\alpha$-SiAlON nas amostras SNYA20 e SNCA20.

[Table III - Refined structural parameters of $\alpha-S i A l O N$ in the SNYA20 and SNCA20 samples.]

\begin{tabular}{ccc}
\hline Parâmetros & SNYA20 & SNCA20 \\
\hline Radiação & $\lambda=1,2398 \AA$ & $\lambda=1,2398 \AA$ \\
Intervalo de $2 \theta$ & $8^{\circ} \leq 2 \theta \leq 78^{\circ}$ & $8^{\circ} \leq 2 \theta \leq 78^{\circ}$ \\
Número de reflexões & 130 & 130 \\
Sistema & hexagonal & hexagonal \\
Grupo Espacial & $P$ 3 1c (no 159) & $P$ 3 1c (n- 159$)$ \\
$a(\AA)$ & $7,8126(1)$ & $7,8138(3)$ \\
$c(\AA)$ & $5,6920(2)$ & $5,6914(3)$ \\
$\mathrm{R}_{\mathrm{p}}$ & 18,5 & 26,4 \\
$\mathrm{R}_{\mathrm{wp}}$ & 20,7 & 27,7 \\
$\mathrm{R}_{\mathrm{e}}$ & 7,30 & 8,81 \\
$\chi^{2}$ & 8,036 & 9,920 \\
\hline
\end{tabular}


a partir de aditivos com diferentes características cristalinas apresentam propriedades estruturais similares.

Os parâmetros de rede refinados dos $\alpha$-SiAlONs sinterizados com $\mathrm{Y}_{2} \mathrm{O}_{3}$ ou $\mathrm{CRE}_{2} \mathrm{O}_{3}$ estão listados na Tabela III. As posições atômicas foram mantidas fixas adotando-se os dados da literatura. Uma dificuldade no refinamento das posições atômicas está no fato de os SiAlONs constituírem uma solução sólida, onde os átomos de $\mathrm{Si}$ e $\mathrm{Al}$ ocupam o mesmo sítio, assim como os átomos de $\mathrm{O}$ e $\mathrm{N}$.

Por outro lado, a constatação de que as posições e as larguras das reflexões de $\alpha$-SiAlON sinterizado com $\mathrm{CRE}_{2} \mathrm{O}_{3}$ apresentam valores similares às de $\alpha$-SiAlON sinterizado com $\mathrm{Y}_{2} \mathrm{O}_{3}$, indicam que os átomos de terras raras devem estar ocupando o mesmo sítio cristalográfico do $\mathrm{Y}$, formando uma solução sólida.

Algumas propriedades físicas e mecânicas determinadas no trabalho anterior [8] mostraram a similaridade das propriedades morfológicas, físicas e mecânicas entre os $\alpha$ SiAlONs preparados a partir da utilização de $\mathrm{Y}_{2} \mathrm{O}_{3}$ ou $\mathrm{CRE}_{2} \mathrm{O}_{3}$. Ambos apresentaram densificação próxima de $98 \%$ e perda de massa de aproximadamente $2 \%$. A microestrutura de ambas as amostras revelaram a presença de grãos alongados com razão de aspecto próxima de 5 e tamanho médio de grãos de aproximadamente $5 \mu \mathrm{m}$. Os resultados das medidas de propriedades mecânicas mostraram valores de dureza em torno de $18,5 \mathrm{GPa}$ e tenacidade à fratura próximos de $5 \mathrm{MPa} \cdot \mathrm{m}^{1 / 2}$. Estes resultados têm demonstrado a possibilidade de substituição do $\mathrm{Y}_{2} \mathrm{O}_{3}$ por $\mathrm{CRE}_{2} \mathrm{O}_{3}$ na produção de $\alpha$-SiAlONs por um custo mais baixo.

\section{CONCLUSÕES}

Cerâmicas baseadas em $\alpha$-SiAlONs foram sinterizados a partir do nitreto de silício utilizando aditivos contendo AlN e $\mathrm{Y}_{2} \mathrm{O}_{3}$ ou um óxido misto formado de ítrio e terras raras $\left(\mathrm{CRE}_{2} \mathrm{O}_{3}\right)$. $\mathrm{O}$ óxido misto é um potencial substituto à ítria comercial, pois possui propriedades físicas e mecânicas similares e é obtido a um custo $80 \%$ inferior, que pode reduzir o preço final do material cerâmico produzido com esse aditivo. Foi comprovada a similaridade nas propriedades estruturais entre os SiAlONs produzidos com ambos aditivos. A similaridade das propriedades estruturais, morfológicas e mecânicas entre SiAlONs produzidos com aditivos contendo $\mathrm{Y}_{2} \mathrm{O}_{3}$ ou $\mathrm{CRE}_{2} \mathrm{O}_{3}$ indicam a possibilidade de substituição do $\mathrm{Y}_{2} \mathrm{O}_{3}$ por $\mathrm{CRE}_{2} \mathrm{O}_{3}$ na produção de $\alpha$-SiAlONs por um custo mais baixo.

\section{AGRADECIMENTOS}

Os autores agradecem ao Laboratório Nacional de Luz Síncrotron (LNLS) pela concessão das facilidades para a realização dos experimentos na linha de luz D12A-XRD1, projeto \#2047/03. C. dos Santos agradece à FAPESP pelo suporte financeiro, processo 01/08682-6.

\section{REFERÊNCIAS}

[1] F. L. Riley, J. Am. Ceram. Soc. 83, 2 (2000) 245.

[2] K. H. Jack, J. Mater. Sci. 11 (1976) 1135.

[3] G. Woetting, B. Kanka, G. Ziegler, in Non-Oxide Technical and Engineering Ceramics. Ed. S. Hampshire. Elsevier, London, UK (1986) pp. 83-96.

[4] M. Mitomo, in Fine Ceramics, Ed. S. Sato, 352p., Elsevier, Tokyo (1985) p.197-205.

[5] S. Kurama, M. Hermann, H. Mandal, J. Eur. Ceram. Soc. 22 (2002) 109.

[6] I. H. Shin, D. J. Kim, Mater. Lett. 47 (2001) 329.

[7] S. Ribeiro, "Estudo comparativo das propriedades do nitreto de silício sinterizado com óxido de ítrio/ óxido de silício e concentrado de óxidos de ítrio e terras raras/óxido de silício", Tese de Doutorado, Faculdade de Engenharia Química de Lorena, Lorena, SP (1997) 194 p.

[8] C. Santos, K. Strecker, S. Ribeiro, J. V. C. Souza, O. M. M. Silva, C. R. M. Silva, Mater. Lett. 58 (2004) 1792.

[9] C. Santos, S. A. Baldacim, O. M. M. Silva, C. R. M., Silva, Int. J. Refract. Metals and Hard Mater. 21, 5-6 (2003) 233.

[10] K. Strecker, R. Gonzaga, S. Ribeiro, M. J. Hoffmann, Mater. Lett. 45 (2000), 39.

[11] C. Santos, K. Strecker, S. Ribeiro, C. R. M. Silva, J. Mater. Proc. Techn. 142-3 (2003) 697.

[12] W. Kraus, G. Nolze, J. Appl. Cryst. 29 (1996) 301.

[13] J. Rodriguez-Carvajal, Reference Guide for the Computer Program FullProf, Laboratoire Leon Brillouin, CEA-CNRS, Saclay, France (1996).

[14] R. W. G. Wyckoff, Crystal structures, Interscience Publishers, (1965) 4 volumes.

[15] P. Villars and L. D. Calvert (Eds.), Pearson's Handbook of Crystallographic Data for Intermetallic Phases, $2^{\text {nd }}$ Ed., ASM International, Materials Park, OH (1991) 4 volumes.

[16] M. Herrmann, S. Kurama, H. Mandal, J. Eur. Ceram. Soc. 22 (2002) 2997.

(Rec. 28/06/2005, Rev. 08/03/2005, Ac. 31/03/2005) 